\title{
Computing vibration-rotation-tunnelling levels of HOD dimer
}

\author{
Xiao-Gang Wang and Tucker Carrington Jr. \\ Chemistry Department, Queen's University, \\ Kingston, Ontario $K^{\gamma} \mathrm{L}$ 3N6, Canada
}

(Dated: March 04, 2014;Aug, 2017)

\begin{abstract}
Using an accurate $6 \mathrm{D}$ water dimer potential energy surface, we compute vibration-rotationtunnelling levels of HOD dimer, by assuming that the two monomers are rigid. HOD dimer has two isomers, a D-bonded isomer and an H-bonded isomer, and the wavefunctions of both isomers have amplitude in four wells. HOD dimer is important because, unlike the case of $\mathrm{H}_{2} \mathrm{O}$ dimer or $\mathrm{D}_{2} \mathrm{O}$ dimer, it is possible to measure the largest tunnelling splitting. Results for HOD dimer, therefore facilitate the testing of $\mathrm{H}_{2} \mathrm{O}$ dimer potentials. In J. Chem. Phys. 102, 1114 (1995), experimental results were interpreted in terms of $1 \mathrm{D}$ models. Experimental splittings of both isomers, obtained by fitting an energy level equation to spectra, are in good agreement with those we compute.
\end{abstract}

*Electronic address: xgwang.dalian@gmail.com

${ }^{\dagger}$ Electronic address: Tucker.Carrington@queensu.ca, Fax: 613-533-6669 


\section{INTRODUCTION}

Water dimer has been studied for decades by both experimentalists [1-12] and theorists [13 28]. It is hoped that understanding the structure of the water dimer energy levels and determining an accurate water dimer potential energy surface (PES) will aid in modelling the effect of hydrogen bonds in biological molecules and chemical reactions in condensed phases. Good models for bulk water are built on the potential energy surface (PES) of water dimer. The water dimer itself is thought to play an important role in Earths atmosphere. The water dimer PES is far from harmonic. It has 8 accessible (by feasible permutationinversion operations) identical wells separated by low barriers. As a consequence, tunnelling is important and the dynamics is complicated. Although it is possible to identify paths between the minimum (and barrier heights along these paths provide valuable information), it is very difficult to develop useful low-dimensional models. For example, the usefulness of 1D models is limited by coupling between vibrational coordinates and by the difficulty of choosing the mass to use in the 1D kinetic energy operator. In this paper, we present results of calculations on HOD dimer done by assuming that the monomers are rigid, but including all six of the inter-monomer coordinates. Rigid monomer calculations are quite accurate, when the monomers are not excited, for HF dimer [29] and water dimer [16-19, 28].

The isotopologue obtained by replacing two protons with deuterons has four isomers. The two that can be described as HOD dimer are shown in Fig. 1. The two that can be described as $\mathrm{H}_{2} \mathrm{O}-\mathrm{D}_{2} \mathrm{O}$ are shown in Fig. 2. No feasible operations convert the isomers in Fig. 1 (2) to isomers in Fig. 2 (1). The isotopologue $\left(\mathrm{H}_{2} \mathrm{O}-\mathrm{HDO}\right)$ obtained by replacing one proton with a deuteron has three isomers, shown in Fig. 3. The isotopologue $\left(\mathrm{D}_{2} \mathrm{O}-\mathrm{HDO}\right)$ obtained by replacing three protons with deuterons has three isomers, shown in Fig. 4. In this paper, we study HOD dimer (Fig. 1) which has an HDO-DOH (D-bonded isomer) and

an HDO-HOD (H-bonded isomer). The atom immediately after the hyphen indicates the $\mathrm{H} / \mathrm{D}$ atom forming the hydrogen bond with the $\mathrm{O}$ atom.

In $\mathrm{H}_{2} \mathrm{O}$ dimer, tunnelling among the 8 wells splits each ro-vibrational level into six levels. The six levels consist of two triple forks. The upper (lower) prong of each triple fork is a $B(A)$ or an $A(B)$ level. The middle prong is an $E$ state. The spectrum is therefore characterised by three splittings. [16, 17] The largest splitting is the difference between the average energy 
of the upper and lower prongs of the top fork and the average energy of the upper and lower prongs of the bottom fork. This largest splitting is called the acceptor switch (AS) splitting. The second largest splitting is the difference between the top and bottom prongs of a fork. This splitting is called the interchange splitting. The third type of splitting, often called the bifurcation splitting is very small. Tunnelling along the AS path does not break the hydrogen bond and the AS path therefore has the lowest barrier.

Due to its importance, there are several good PESs for $\left(\mathrm{H}_{2} \mathrm{O}\right)_{2}$. According to the BornOppenheimer approximation, a PES for $\left(\mathrm{H}_{2} \mathrm{O}\right)_{2}$ should also be valid for $(\mathrm{HOD})_{2}$. To verify the accuracy of a PES, it is always a good idea to confirm that spectra computed for all isotopologues agree well with experimental data. For $\left(\mathrm{H}_{2} \mathrm{O}\right)_{2}$ this is particularly important because the AS splitting cannot be measured. Transitions between the $A$ (or $B$ ) level in the bottom fork and the $A$ (or $B$ ) level in the top fork are forbidden by symmetry. Although transitions from the $E$ level of the bottom fork to the $E$ level of the top fork are in principle possible, they have never been measured due to their weak intensities. See however Ref. 9 , Only the sum of the AS tunnelling splitting for $K=0$ and $K=1$ has been measured. $K$ is the quantum number for the projection of the total angular momentum on the inter-monomer axis, $-J \leq K \leq J$.

Each of the two HOD dimer isomers has four accessible wells. Each ro-vibrational level is therefore split into four states. HOD dimer has only two types of tunnelling splittings: the AS and interchange splittings. There is no bifurcation splitting because the $\mathrm{H}$ and $\mathrm{D}$ nuclei have different masses. As is the case for $\left(\mathrm{H}_{2} \mathrm{O}\right)_{2}$, the larger (AS) tunnelling splitting arises from a motion which interchanges the $\mathrm{H}$ and $\mathrm{D}$ on the acceptor monomer. This tunnelling is called methyl-amine-type internal-rotation motion in Refs. 30, 31. The smaller tunnelling splitting arises from the interchange of the donor and acceptor roles of the two monomers. In HOD dimer it is possible to measure the AS splitting for a single value of $K$. This makes it possible to compare theoretical AS tunnelling splittings with experimental values, thereby testing the accuracy of the PES. Experimental values for $K=0$ and $K=1$ are known for both isomers of HOD dimer [30].

Since no transitions between levels of different isomers were found, there is nothing known experimentally about their relative energies. Our calculation shows that the ground state of D-bonded isomer is lower than that of the H-bonded isomer by $57.37 \mathrm{~cm}^{-1}$. 


\section{EVALUATING THE PES IN HOD DIMER COORDINATES}

According to the Born-Oppenheimer approximation, one can calculate energy levels of HOD dimer using an $\left(\mathrm{H}_{2} \mathrm{O}\right)_{2}$ PES. To do this, we must evaluate the $\left(\mathrm{H}_{2} \mathrm{O}\right)_{2}$ PES at values of the internal coordinates used for HOD dimer. We compute the spectra of HOD dimer assuming the monomers are rigid. The coordinates we use are denoted $\left(\alpha, \beta ; \Omega_{A} ; \Omega_{B} ; r_{0}\right)$. 32 . See Fig. 5. The orientation of the molecule-fixed (MF) frame of monomer $X$ with respect to the dimer-fixed (DF) frame attached to the vector from the centre of mass of monomer A to the centre of mass of monomer $\mathrm{B}, \overrightarrow{r_{0}}$, is specified by the Euler angles $\Omega_{X}, X=\mathrm{A}, \mathrm{B} . r_{0}$ is the length of $\overrightarrow{r_{0}}$, the vector from the centre of mass of monomer $\mathrm{A}$ to the centre of mass of monomer B. $\alpha$ and $\beta$ specify the orientation of $\overrightarrow{r_{0}}$ with respect to a space-fixed (SF) frame. The axes of the MF frames are the principal axes of the monomers. We use the 6D water dimer PES called CCpol-8s[19] in the literature. Its input is the internal coordinates of $\left(\mathrm{H}_{2} \mathrm{O}\right)_{2}$, denoted in this paper $\left(\Omega_{A}^{\prime} ; \Omega_{B}^{\prime} ; r_{0}^{\prime}\right)$. When $\left(\mathrm{H}_{2} \mathrm{O}\right)_{2}$ and $(\mathrm{HOD})_{2}$ have the same shape they have the same potential value, however, $\left(\Omega_{A} ; \Omega_{B} ; r_{0}\right)$ and $\left(\Omega_{A}^{\prime} ; \Omega_{B}^{\prime} ; r_{0}^{\prime}\right)$ are different, because the coordinates are mass dependent. One must therefore convert $\left(\Omega_{A} ; \Omega_{B} ; r_{0}\right)$ to $\left(\Omega_{A}^{\prime} ; \Omega_{B}^{\prime} ; r_{0}^{\prime}\right)$ so that the potential can be evaluated.

To explain how this is done we refer to $(\mathrm{HOD})_{2}$ as the minor isotopologue and $\left(\mathrm{H}_{2} \mathrm{O}\right)_{2}$

as the major isotopologue. We first (i) convert $\left(\Omega_{A} ; \Omega_{B} ; r_{0}\right)$ to Cartesian coordinates in the DF frame of the minor isotopologue and then (ii) convert these Cartesian coordinates to $\left(\Omega_{A}^{\prime} ; \Omega_{B}^{\prime} ; r_{0}^{\prime}\right)$. Step (ii) would not be required if the input to the PES were Cartesian coordinates (as is sometimes the case, e.g. in the PESs HBB2[33], WHBB[26], MB-pol[27]).

The DF frame Cartesian coordinates (step (i)), of atom $i$ in monomer $A, \boldsymbol{\xi}_{A i}^{\mathrm{DF}}$ are obtained from the MF frame Cartesian coordinates by a rotation

$$
\begin{aligned}
\boldsymbol{\xi}_{A i}^{\mathrm{DF}} & =\boldsymbol{S}^{t}\left(\alpha_{A}, \beta_{A}, \gamma_{A}\right) \boldsymbol{\xi}_{A i}^{\mathrm{MF}} \\
\boldsymbol{\xi}_{B i}^{\mathrm{DF}} & =\boldsymbol{S}^{t}\left(\alpha_{B}, \beta_{B}, \gamma_{B}\right) \boldsymbol{\xi}_{B i}^{\mathrm{MF}}+\left(0,0, r_{0}\right)^{t}
\end{aligned}
$$

where $\boldsymbol{S}$ is the direction cosine matrix defined in Ref. 34, $\boldsymbol{\xi}_{A i}^{\mathrm{MF}}$ are the Cartesian coordinates of atom $i$ of minor isotopologue monomer in the MF frame. They are easily calculated once the frame is known. The MF frames are principal-axis frames for the the rigid monomers. Note that the orientation of the minor MF frame is tilted with respect to the orientation of the bisector frame (which is the same as the principal axes frame for $\mathrm{H}_{2} \mathrm{O}$ ). See Fig. 6. 
Eq. (1) could also be used if the monomers were not rigid.

Knowing the DF frame minor Cartesian coordinates for a particular shape, we wish to find the corresponding major internal coordinates (step (ii)). To do this we first determine the orientation of the major MF frames. The major MF frames are bisector frames. For example, for monomer $A$, the unit vectors are

$$
\begin{aligned}
\hat{e}_{z}^{\mathrm{MF}} & =-c\left(b_{2} \vec{b}_{1}+b_{1} \vec{b}_{2}\right) \\
\hat{e}_{y}^{\mathrm{MF}} & =\frac{1}{b_{1} b_{2} \sin \theta} \vec{b}_{1} \times \vec{b}_{2} \\
\hat{e}_{x}^{\mathrm{MF}} & =\hat{e}_{y}^{\mathrm{MF}} \times \hat{e}_{z}^{\mathrm{MF}},
\end{aligned}
$$

where, $\vec{b}_{i}=\boldsymbol{\xi}_{i}^{\mathrm{DF}}-\boldsymbol{\xi}_{3}^{\mathrm{DF}}$ is a bond vector for $\mathrm{OH}_{i}$ for monomer $\mathrm{A}$ and $c$ is a positive normalization constant. $\theta$ is the angle between $\vec{b}_{1}$ and $\vec{b}_{2}$. Even though these unit vectors are for the major isotopologue, there is no need to attach a prime superscript since they are clearly defined in Eq. (2). We also need, $\overrightarrow{r_{0}^{\prime}}$, the vector pointing from the centre of mass of monomer $A$ to that of monomer $B$. From the MF frame unit vectors and vector $\overrightarrow{r_{0}^{\prime}}$, one can determine the last two Euler angles for both monomers ( $A$ and $B$ labels are not indicated),

$$
\begin{aligned}
& \beta^{\prime}=\arccos \left(\frac{1}{r_{0}^{\prime}} \overrightarrow{r_{0}^{\prime}} \cdot \hat{e}_{z}^{\mathrm{MF}}\right) \\
& \gamma^{\prime}=\pi-\arctan \left(\frac{\overrightarrow{r_{0}^{\prime}} \cdot \hat{e}_{y}^{\mathrm{MF}}}{\overrightarrow{r_{0}^{\prime}} \cdot \hat{e}_{x}^{\mathrm{MF}}}\right) .
\end{aligned}
$$

These equations are obtained by recognizing that the spherical polar angles of $\overrightarrow{r_{0}^{\prime}}$ in the MF frame are $\left(\beta^{\prime}, \pi-\gamma^{\prime}\right)$. They are equivalent to $\left(-\beta^{\prime},-\gamma^{\prime}\right)$, but we use $\left(\beta^{\prime}, \pi-\gamma^{\prime}\right)$ because the polar angle cannot be negative. It remains to find $\alpha_{B}^{\prime}-\alpha_{A}^{\prime}$. This is done via intermediate (one for each monomer) frames, labelled by "int", which are obtained by rotating the dimerfixed frame about its $z$-axis by $\alpha^{\prime}$. Both the intermediate frames have the same $z$ axis. Unit vectors for the intermediate frames can be obtained from the unit vectors of the MF frames (Eqs. (2)), by recognizing that by rotating an intermediate frame about its $y$-axis by $\beta^{\prime}$ and then about its $z$-axis by $\gamma^{\prime}$ where $\beta^{\prime}$ and $\gamma^{\prime}$ are given in Eq. (3) one obtains a MF frame. Therefore, the $x$ unit vector of an intermediate frame is

$$
\hat{e}_{x}^{\mathrm{int}}=S_{1,1}\left(0, \beta^{\prime}, \gamma^{\prime}\right) \hat{e}_{x}^{\mathrm{MF}}+S_{2,1}\left(0, \beta^{\prime}, \gamma^{\prime}\right) \hat{e}_{y}^{\mathrm{MF}}+S_{3,1}\left(0, \beta^{\prime}, \gamma^{\prime}\right) \hat{e}_{z}^{\mathrm{MF}}
$$

The dihedral angle between the plane that contains the $z$ axis and the $x$ axis of the $A$ 
intermediate frame and the plane that contains the $z$ axis and the $x$ axis of the $B$ intermediate frame is $\alpha_{B}^{\prime}-\alpha_{A}^{\prime}$.

Some water dimer PESs (such as SAPT-5st[17]) depend on Cartesian coordinates of sites attached to the monomers. For example, in the SAPT-5st case, from the $\mathrm{H}_{2} \mathrm{O}$ dimer PES code, it is straightforward to make a code for the $\mathrm{D}_{2} \mathrm{O}$ dimer PES by recomputing the position of the 5 sites. This is done by shifting the origin of the Cartesian axes from the centre of mass of $\mathrm{D}_{2} \mathrm{O}$ to the centre of mass of $\mathrm{H}_{2} \mathrm{O}$ (a simple shift along the $C_{2}$ axis). In fact the authors of the SAPT-5st PES provide $\mathrm{H}_{2} \mathrm{O}$ dimer and $\mathrm{D}_{2} \mathrm{O}$ dimer options. We use their $\mathrm{D}_{2} \mathrm{O}$ dimer option to test our coordinate transformation. Values of their $\mathrm{D}_{2} \mathrm{O}$ dimer PES and values we obtain by transforming the internal coordinates of $\mathrm{D}_{2} \mathrm{O}_{-} \mathrm{D}_{2} \mathrm{O}$ to the internal coordinates of $\mathrm{H}_{2} \mathrm{O}-\mathrm{H}_{2} \mathrm{O}$ agree. We also compute ro-vibrational energy levels with both approaches and they agree as well.

\section{CALCULATION DETAILS}

We use the kinetic energy operator of Brocks et al. [32] and the CCpol-8s[19] PES. Eigenvalues and eigenvectors of a basis representation of the Hamiltonian operator are computed with the Lanczos algorithm. Quadrature is used for potential matrix elements and matrixvector products are evaluated by doing sums sequentially. [35 38] To do the calculation we must know (for the kinetic energy operator) the rotational constants of HOD and (to evaluate the PES) the orientation of the MF frames. The rotational constants for HDO are taken to be the experimental values [39], $A=23.41911, B=6.40655$, and $C=9.10311 \mathrm{~cm}^{-1}$. The MF frames are principal axis frames. To find the orientation of the principal axes we need the tilt angle referred to in Section II. To get the tilt angle, it would be ideal to use the vibrational ground state averaged geometry of HOD. This, however, is inconsistent with the 6D PES which is constructed assuming the geometry of the monomers is the ground state averaged geometry of $\mathrm{H}_{2} \mathrm{O}$. To determine the tilt angle, we therefore use the vibrational ground state averaged geometry of $\mathrm{H}_{2} \mathrm{O}$, but when computing the moments and products of inertia we replace one of the $\mathrm{H}$ with $\mathrm{D}$. The $\mathrm{H}_{2} \mathrm{O}$ equilibrium structure we use is $r(\mathrm{O}-\mathrm{H})=$ $0.9716257 \AA, \theta(\mathrm{H}-\mathrm{O}-\mathrm{H})=104.69^{\circ}$. [17] The tilt angle is $21.0^{\circ}$. See Fig. 6 . 


\section{A. Basis}

We choose to use the most accurate 6D $\left(\mathrm{H}_{2} \mathrm{O}\right)_{2}$ PES, CCpol-8s[19]. 6D $\left(\mathrm{H}_{2} \mathrm{O}\right)_{2}$ vibrationrotation-tunnelling (VRT) levels have been computed previously [19, 24, 28]. The 6D HOD dimer calculations are done with the parity-adapted ro-vibrational basis functions of Ref. 28. They are linear combinations of products of three symmetric-top eigenfunctions. The monomer symmetric-top eigenfunctions are labelled by $j_{X}, m_{X}, k_{X}, X=A, B$. For the calculations of this paper, the maximum value of $j_{X}, m_{X}, k_{X}$ is $14 . N_{r_{0}}=9$ is the number of PODVR (potential optimised discrete variable representation) points. The numbers of angular quadrature points are $N_{\theta}=15$ (for $\beta_{A}, \beta_{B}$ ) and $N_{\phi}=30$ (for $\alpha_{A}-\alpha_{B}, \gamma_{A}, \gamma_{B}$ ). The same PODVR basis [40, 41] was used in the 6D calculation of $\mathrm{H}_{2} \mathrm{O}$ dimer in Ref. 28 where it was shown that levels up to $150 \mathrm{~cm}^{-1}$ are converged to within $0.001 \mathrm{~cm}^{-1}$. The PODVR functions are calculated using a basis of 200 sine functions in a box from 4.2 to 10.0 Bohr for $r_{0}$. We use the $G_{4}$ permutation-inversion group in the calculation. The permutation-inversion group consists of 4 symmetry operations: $\left\{E, E^{*}\right\} \otimes\left\{E, P_{a b}\right\}$ where $E^{*}$ is the inversion operation and $P_{a b}$ permutes the two monomers. $G_{4}$ has four symmetry labels: $A^{+}, A^{-}, B^{+}$, and $B^{-}$. $+/-$ label even/odd symmetry under $E^{*}$ and $A / B$ label symmetric/antisymmetric irreps under $P_{a b}$.

To define the PODVR basis, we need to know the equilibrium structure. It was determined by finding the values of the HOD dimer coordinates that minimize the potential (using the coordinate transformation of section II). The minima for the D-bonded and H-bonded isomers have the same energy. The $r_{0}$ reference potential used to define the PODVR basis is the cut potential for which the other coordinates have their values at the D-bonded isomer minimum (the cut potential for which the other coordinates have their values at the $\mathrm{H}$-bonded isomer minimum should be very similar). The equilibrium structures of the D-bonded and H-bonded isomers of HOD dimer on the CCpol-8s PES are given in Table If. The minimum energy is $-1785.16 \mathrm{~cm}^{-1}$, the same as the minimum energy of $\mathrm{H}_{2} \mathrm{O}$ dimer. Compared with the equilibrium structure of the $\mathrm{H}_{2} \mathrm{O}$ on the same PES: $\left(\alpha_{A}, \beta_{A}, \gamma_{A} ; \alpha_{B}, \beta_{B}, \gamma_{B}\right)=\left(0.0^{\circ}, 55.90^{\circ}, 90.0^{\circ} ; 180^{\circ}, 60.55^{\circ}, 0^{\circ}\right)$, and $r_{0 e}=5.5045 a_{0}$, the biggest difference is in $\beta_{B}$ (the angle associated with the donor). $\beta_{B}$ increases by roughly $20^{\circ}$ for the D-bonded isomer and decreases by roughly $20^{\circ}$ for the H-bonded isomer. This 
change is due largely to the $z$ principal axis of the HDO monomer being tilted by $21.0^{\circ}$ from the $z$ principal axis of the $\mathrm{H}_{2} \mathrm{O}$ monomer, shown in Fig. 6. The D-bonded isomer has a smaller $r_{0 e}$ simply because the centre of mass of the donor is closer to the acceptor than in the H-bonded isomer. The biggest difference between the coordinates of the H-bonded and D-bonded isomers is the $\gamma_{B}$ value. The D-bonded value is about $\pi$ larger than its H-bonded counterpart, i.e., the donor, on the right, is rotated by $\pi$ about the $z$-axis of its MF frame.

\section{B. Distinguishing D-bonded and H-bonded isomers}

The D-bonded and H-bonded isomers of HOD dimer correspond to two different sets of four wells on the same PES. We calculate solutions of the Schroedinger equation on the PES and therefore obtain both wavefunctions localized in the D-bonded wells and in the H-bonded wells. To label states as being D-bonded or H-bonded, we examine wavefunctions. States localized in the D-bonded and H-bonded wells have different coordinate ranges. The appropriate coordinate ranges are apparent from the equilibrium structures of the two isomers (Table I). The largest difference between the two structures is the value of the last Euler angle of the donor: $\gamma_{D}$ ( or $\gamma_{B}$ if we put the donor on the right and label it as B). The D-bonded isomer has $\gamma_{B}$ values near $180^{\circ}$. The H-bonded isomer has $\gamma_{B}$ values near $0^{\circ}$. By rotating the donor by $180^{\circ}$ about its $C_{2}$ axis, one moves to the H-bonded well from D-bonded well. The two isomers also have somewhat different values of $\beta_{B}: 82^{\circ}$ and $41^{\circ}$. This is due to the tilt of the HOD MF frame. The difference between them is about twice the value of the tilt angle. The simplest way to distinguish the isomers is to fix all other coordinates and examine wavefunctions as a function of $\gamma_{B}$. The exact values used for the other coordinates is not important; we use averages for the equilibrium geometries of the two isomers: $\beta_{A}=58^{\circ}, \gamma_{A}=105^{\circ}, \beta_{B}=61^{\circ}$, and $r_{0}=5.44$ Bohr. In Fig. 7, we plot

$2 \mathrm{D}$ cuts $\Psi_{\alpha_{A}-\alpha_{B}, \gamma_{B}}$ of selected states to demonstrate how we identify the two isomers. For the rotational coordinates, arbitrary values $\alpha=\beta=45^{\circ}$ are chosen. Once we have sorted the levels into two groups, one for H-bonded and one for D-bonded, we find that the energy levels of both isomers have the same symmetry pattern. 


\section{RESULTS AND DISCUSSION}

The ground state energy level structures of the D-bonded and H-bonded isomers are the same and shown in Fig. 8 and Fig. 9, respectively. Each rotational level is split into four levels by tunnelling. Following Fig. 1 of Karyakin et al [30], the four sub-levels for $K=0$ are labelled as $V=0,1,2$, and 3, and the four sub-levels for $|K|=1$ are labelled as $V=4,5,6$, and 7. $V$ labels a group of levels with different $J$ and the same $|K|$. Each level is labelled by $(V, J, K)$. Following Karyakin et al., when $|K|>0$ and two levels with the same $|K|$ are nearly degenerate, the lower of the two is labelled with $K=-|K|$. We assign $|K|$ to each of our levels by analysing the calculated wavefunctions. 42] In their figure, Karyakin et al[30] show only $J=1$ levels. Fig. 8 includes also $J=0$ levels. This makes it easier to visualize transitions. The symmetry labels of Fig. 8 (obtained from the symmetryadapted Lanczos method [43, 44]) match perfectly those of Fig. 4 of the effective tunnelling Hamiltonian model (internal-axis-method, IAM) of Coudert and Hougen. [45]. The dipole transition selection rule is $A^{+} \leftrightarrow A^{-}$and $B^{+} \leftrightarrow B^{-}$.

For HOD dimer Karyakin et al. determine splittings by choosing the parameters of the simple energy level expression

$$
E(J, K, V)=E_{V}+\bar{B}_{V}\left[J(J+1)-K^{2}\right]-D_{V}\left[J(J+1)-K^{2}\right]^{2}+\left((B-C)_{V} / 4\right) K J(J+1)
$$

so that differences between different $E(J, K, V)$ reproduce their experimental transition frequencies. $E_{V}$ is the origin for the group of levels labelled by $V$. Note that $K$ may be positive or negative. Two nearly degenerate levels labelled by $+K$ and $-K$ have the same $E_{V}$.

For $\mathrm{H}_{2} \mathrm{O}$ dimer and $\mathrm{D}_{2} \mathrm{O}$ dimer, only a- and c-type transitions are observed. The b-type transitions are absent because the component of the dipole perpendicular to the $C_{s}$ symmetry plane is zero. The absence of $b$-type transitions prevents the determination of AS tunnelling splittings, denoted $a(K)$. For both the D-bonded and the H-bonded isomers of HOD dimer, a-, b- and c-type transitions between $V$ levels are observed. [30] For each $J$, there are four symmetry-allowed b-type and four symmetry-allowed c-type transitions between $K=0$ and $|K|=1$ levels. All of them are observed by Karyakin et al, but some low- $J$ lines are not seen. Because b-type and c-type transitions are both present, it is possible to observe two transitions, between different $V$ states in different forks, which have either identical initial or identical final $V$ states. For example, the $V=0 \rightarrow 5$ b-type line and $V=0 \rightarrow 7$ c-type line 
provide information about the spacing between $V=5$ and $V=7$. If all transitions were from upper fork to upper fork or from lower fork to lower fork, it would be impossible to determine $a(K)$. Some $J \leq 1$ transition pairs that contain information about AS splittings are not observed. For the D-bonded isomer, there is only one pair: a b-type transition between $V=3$ and 6 at $104.828 \mathrm{GHz}$, and a c-type transition between $V=1$ and 6 at 222.054 $\mathrm{GHz}$, marked in Fig. 8. The difference between these transitions determines the difference between $V=3$ and $V=1$ levels. After Karyakin et al. fit Eq. (5) to all the assigned levels, they use it to determine the AS splittings from $E_{V}$. Although in principle $a(K)$ depends on $J$, they find one set of $a(K)$ from transitions between many $J$ values. Karyakin et al. use

$$
\begin{aligned}
& a(0)=1 / 2\left(E_{2}+E_{3}\right)-1 / 2\left(E_{0}+E_{1}\right) \\
& a(1)=1 / 2\left(E_{6}+E_{7}\right)-1 / 2\left(E_{4}+E_{5}\right) .
\end{aligned}
$$

A much simpler way to get $a(0)$ would be to measure transitions between the two forks for $K=0$. Such transitions are allowed by symmetry However, they are forbidden if $K$ is a good quantum number because there are no $\mathrm{K}=0$ Q-branch lines. Due to Coriolis coupling between $K=0$ and $K=1$ levels (marked by two wavy red lines in Fig. 8), Karyakin et al. [30] were able to observe two such forbidden lines: $\mathrm{V}=1 \rightarrow 2,4_{04} \rightarrow 4_{04}$, and $\mathrm{V}=0 \rightarrow$ $3,4_{04} \rightarrow 4_{04}$, where we use the standard $J_{K_{a} K_{c}}$ notation [34] to label a ro-vibrational state.

Since we have computed all the VRT levels with $J \leq 1$, unlike the experimentalists, we can calculate the AS splittings simply from energy level differences. This, of course, will give us $J$ dependent $a(K)$. We choose four tunnelling levels for each $J_{K_{a}, K_{c}}$ and compute AS splitting from equations like Eq. (6), but with $E_{V}$ replaced with actual energy levels. As shown by the double arrows in Fig. 8, we can compute $a(K=0)$ from both $J=0$ and $=$ $J=1$ levels. The two $a(0)$ are very close and both can be compared to the experimental $a(0)$ derived by fitting. By the same token, we can compute $a(1)$ from either the $K=+1$ or the $K=-1$ levels as as shown by double arrows in Fig. 8, $a(+1)$ and $a(-1)$ can be compared to the experimental $a(1)$ derived by fitting. We could also, like the experimentalists fit using the same energy level expression the experimentalists used. The disadvantage of this fitting approach is that we would need to compute high $J$ levels in order to get meaningful fitting constants.

The AS tunnelling splittings for the D-bonded isomer of HOD dimer are given in Table 
IV. For $a(0)$, the calculated $\left(7.88 \mathrm{~cm}^{-1}\right)$ and experimental $\left(7.15 \mathrm{~cm}^{-1}\right)$ values are in good agreement. For $a(1)$, the calculated $\left(4.37 \mathrm{~cm}^{-1}\right)$ and experimental $\left(4.04 \mathrm{~cm}^{-1}\right)$ values are also close. Although their absolute errors are rather different, the AS tunnelling splittings for $K=0$ and $K=1$ both have about $10 \%$ relative error on the CCpol-8s PES which is the most accurate $6 \mathrm{D}$ PES. In comparison, for $\mathrm{H}_{2} \mathrm{O}$ dimer, only $a(0)+a(1)$ is measured and its relative error is also about $10 \%$ : $13.92 \mathrm{~cm}^{-1}$ from experiment (see Ref. 19 and references therein) and $15.33 \mathrm{~cm}^{-1}$ from theory. For $\mathrm{D}_{2} \mathrm{O}$ dimer, the experimental value of $a(0)+a(1)$ is $2.39 \mathrm{~cm}^{-1}$ (see Ref. 19 and references therein)) and the theoretical value is $2.68 \mathrm{~cm}^{-1}$, again the relative error is about $10 \%$. For these different isotopomers and $K$ values, the relative error in $a(K)$ is always about $10 \%$. We note that Saykally and coworkers have estimated $a(0)=9.33 \mathrm{~cm}^{-1}\left[46\right.$ ] for $\mathrm{H}_{2} \mathrm{O}$ dimer and $1.77 \mathrm{~cm}^{-1}\left[\right.$ [ ] for $\mathrm{D}_{2} \mathrm{O}$ dimer from experimental data (see e.g. Ref. [8), but using the assumption that the AS splitting for the ground state and excited state (antisymmetric stretch vibrational excited state of the acceptor) are equal. This assumption is likely to lead to significant error and the estimated $a(0)$ should not be considered an experimental value (see the explanation in Ref. 8). The AS tunnelling splittings for the H-bonded isomer of HOD dimer are given in Table VI. Again the relative errors are about $10 \%$.

One expects $a(0)$ calculated from $J=0$ levels to be very close to $a(0)$ calculated from $J=1$ levels. When this is not the case, it is because some levels are shifted by coupling. The wavy red lines in Fig. 8 join levels that are close and share the same symmetry. They have different $K$ values and appear to be shifted due to Coriolis coupling. In their fit, the authors of Ref. 30 find they need to introduce two parameters to account for this coupling for the D-bonded isomer, but not for the H-bonded isomer. In our results, the coupling manifests itself in the difference between $a(0)$ values for $J=0$ and $J=1$. For the D-bonded isomer (see Table IV) the two $a(0)$ are 7.882 and $7.888 \mathrm{~cm}^{-1}$. For the H-bonded isomer (see Table VI), the two $a(0)$ are 4.508 and $4.507 \mathrm{~cm}^{-1}$. The smaller difference between the two $a(0)$ for the H-bonded isomer indicates that for it the coupling is less effective, consistent with the finding of Ref. 30.

Interchange tunnelling splittings, $i_{V, V^{\prime}}$ were calculated by Karyakin et al. [30] from differences of $E_{V}$ values, i.e. $i_{V, V^{\prime}}=E_{V^{\prime}}-E_{V}$. We compute them directly from the calculated levels. The results for the D-bonded and H-bonded isomer of HOD dimer are given in Table 
$\mathrm{V}$ and VII respectively. The theoretical and experimental numbers are very close. The variations of the interchange tunnelling splittings between $K=0$ and $K=1$, and between the lower fork and the higher fork with the same $K$ all agree well. Karyakin et al. [30] could not explain these variations in terms of geared and anti-geared pathways for this tunnelling motion and suggested that this failure of the 1D models might be due to the coupling of this interchange tunnelling motion with AS tunnelling motion.

\section{CONCLUSION}

Water dimer has been studied for more than 40 years. [13] Its spectrum is complicated because there are many equivalent wells separated by low barriers. Although it is possible to identify low-lying paths between the wells, 1D models are of limited value, due to coupling and to uncertainly about what mass to use in the 1D KEO. There are many comparisons of experimental and 6D calculated spectra. [19] To thoroughly test a PES, it is important to do calculations on several isotopologues. In this paper we test the best rigid monomer PES of $\left(\mathrm{H}_{2} \mathrm{O}\right)_{2}$ [19] by computing, for the first time, the spectrum of $(\mathrm{HOD})_{2}$.

The HOD dimer calculation is especially important because whereas for $(\mathrm{HOD})_{2}$ it is possible to measure the largest tunnelling splitting (the AS tunnelling) $a(K)$ for $K=0$ and $K=1$, it is impossible to measure $a(0)$ and $a(1)$ for $\left(\mathrm{H}_{2} \mathrm{O}\right)_{2}$. For $\left(\mathrm{H}_{2} \mathrm{O}\right)_{2}$ only the sum $a(0)+a(1)$ can be measured. We find that for the $\mathrm{D}$-bonded isomer, the experimental and calculated AS tunnelling splittings are $7.15 \mathrm{~cm}^{-1}$ and $7.88 \mathrm{~cm}^{-1}$ for $a(0)$, and $4.04 \mathrm{~cm}^{-1}$ and $4.37 \mathrm{~cm}^{-1}$ for $a(1)$. We find that for the H-bonded isomer, the experimental and calculated AS tunnelling splitting are $3.92 \mathrm{~cm}^{-1}$ and $4.51 \mathrm{~cm}^{-1}$ for $a(0)$, and $1.52 \mathrm{~cm}^{-1}$ and $1.68 \mathrm{~cm}^{-1}$ for $a(1)$. Both the $K=0$ and $K=1$ AS tunnelling splittings have relative errors of about $10 \%$, for both D-bonded and H-bonded isomer. In comparison, the relative error in the sum $a(0)+a(1)$ for both $\left(\mathrm{H}_{2} \mathrm{O}\right)_{2}$ and $\left(\mathrm{D}_{2} \mathrm{O}\right)_{2}$ is also about $10 \%$. On the basis of our results for HOD dimer, it seems likely that, for $\left(\mathrm{H}_{2} \mathrm{O}\right)_{2}$ and $\left(\mathrm{D}_{2} \mathrm{O}\right)_{2}$, the relative errors in the individual $a(0)$ and $a(1)$ are also similar. If this is true, then using the theoretical values in Ref. 28, one can estimate the the unobservable $a(0)$ and $a(1)$ for $\left(\mathrm{H}_{2} \mathrm{O}\right)_{2}$ and $\left(\mathrm{D}_{2} \mathrm{O}\right)_{2}$. The smaller interchange tunnelling splittings have similar relative errors.

There are two HOD dimer isomers, the D-bonded isomer and the H-bonded isomer. 
Although the minimum potential values of both isomers are equal, their ground state energies are different. The D-bonded isomer ground state energy is $-1199.24 \mathrm{~cm}^{-1}$ (the potential minimum is at $-1785.16 \mathrm{~cm}^{-1}$ ) and the H-bonded isomer ground state energy is $57.37 \mathrm{~cm}^{-1}$ higher than the D-bonded isomer ground state energy. In comparison, the ground sate energy of $\mathrm{H}_{2} \mathrm{O}$ dimer on the same PES is $-1094.23 \mathrm{~cm}^{-1}$ [28], higher than both isomers of HOD dimer because of the smaller masses of $\mathrm{H}_{2} \mathrm{O}$ dimer. We find that the energy level patterns of both isomers are similar.

[1] T.R. Dyke, K.M. Mack, J. S., Muenter, J. Chem. Phys. 66, 498 (1997)

[2] E.N. Karyakin, G.T. Fraser, F. J. Lovas, R.D. Suenram, M. J. Fujitake, J. Chem. Phys. 102, $1114(1995)$

[3] K.L. Busarow, R.C. Cohen, G.A. Blake, K. B. Laughlin, Y. T. Lee, R. J. Saykally, J. Chem. Phys. 90, 3937 (1989)

[4] L.B. Braly, K. Liu, M. G. Brown, F. N. Keutsch, R. S. Fellers, R.J. Saykally, J. Chem. Phys. $112,10314(2000)$

[5] N. Pugliano, J. D. Cruzan, J. G. Loeser, R. J. Saykally, J. Chem. Phys. 98, 6600 (1993)

[6] J. B. Paul, R. A. Provencal, C. Chapo, K. Roth, R. Casaes, and R. J. Saykally, J. Phys. Chem. A, 103, $2972(1999)$

[7] F. N. Keutsch, N. Goldman, H. A. Harker, C. Leforestier, and R. J. Saykally Mol. Phys. 101, 3477 (2003)

[8] H. A. Harker, F. N. Keutsch, C. Leforestier, Y. Scribano, J.-X. Han and R. J. Saykally Mol. Phys. 105, 497 (2007)

[9] H. A. Harker, F. N. Keutsch, C. Leforestier, Y. Scribano, J.-X. Han and R. J. Saykally Mol. Phys. 105, 513 (2007)

[10] B. E. Rocher-Casterline, L. C. Ch'ng, . K. Mollner, and H. Reisler, J. Chem. Phys. 134, $211101(2011)$

[11] W. T. S. Cole, R. S. Fellers, M. R. Viant, C. Leforestier, and R. J. Saykally J. Chem. Phys., 143, 154306 (2015).

[12] A. Mukhopadhyay, W. T.S. Cole, and R. J. Saykally, Chem. Phys. Lett. 633, 13 (2015). 
[13] T. R. Dyke, J. Chem. Phys. 66, 492 (1977)

[14] S. C. Althorpe and D. C. Clary J. Chem. Phys. 101, 3603 (1994).

[15] S. C. Althorpe and D. C. Clary J. Chem. Phys. 102, 4390 (1995).

[16] C. Leforestier, L. B. Braly, K. Liu, M. J. Elroy, and R. J. Saykally, J. Chem. Phys. 106, 8527 (1997)

[17] G. C. Groenenboom, P. E. S. Wormer, A. van der Avoird, E. M. Mas, R. Bukowski, and K. Szalewicz, J. Chem. Phys. 113, 6702 (2000)

[18] M. J. Smit, G. C. Groenenboom, P. E. S. Wormer, and A. van der Avoird, R. Bukowski, and K. Szalewicz, J. Phys. Chem. 105, 6212 (2001).

[19] W. Cencek, K. Szalewicz, C. Leforestier, R. van Harrevelt, and A. van der Avoird, Phys. Chem. Chem. Phys. 10, 4716 (2008).

[20] R. E. A. Kelly, J. Tennyson, G. C. Groenenboom, and A. van der Avoird, J. Quant. Spectrosc. Radiat. Transfer. 111, 1262 (2010).

[21] J. Tennyson, M. J. Barber, and R. E. A. Kelly, Phil. Trans. R. Soc. A 370, 2656 (2012).

[22] J. O. Richardson, S. C. Althorpe, and D. J. Wales J. Chem. Phys. 135, 124109 (2011)

[23] C. Leforestier, F. Gatti, Raymond S. Fellers, and Richard J. Saykally J. Chem. Phys. 117, $8710(2002)$.

[24] C. Leforestier, K. Szalewicz, and A. van der Avoid, J. Chem. Phys. 137, 014305 (2012).

[25] C. Leforestier, Phil. Trans. R. Soc. A 3702675 (2012)

[26] Y. Wang, X. Huang, B. C. Shepler, B. J. Braams, and J. M. Bowman J. Chem. Phys. 134, 094509 (2011)

[27] V. Babin, C. Leforestier, and F. Paesani, J. Chem. Theo. Comp. 9, 5395 (2013).

[28] X.-G. Wang and T. Carrington, Jr., J. Chem. Phys. 148, 074108 (2018).

[29] Dong H. Zhang, Qian Wu, John Z. H. Zhang, Michael von Dirke, and Zlatko Bacic, Journal of Chemical Physics 102, 2315 (1995)

[30] E. N. Karyakin, G. T. Fraser, F. J. Lovas, R. D. Suenram, and M. Fujitake, J. Chem. Phys. 122, 1114 (1995).

[31] G. T. Fraser, F. J. Lovas, R. D. Suenram, E. N. Karyakin, A. Grushow, W. A. Burns, and K. R. Leopold, J. Mol. Spectrosc. 181, 229 (1997).

[32] G. Brocks, A. Van Der Avoird, B. T. Sutcliffe, and J. Tennyson, Mol. Phys. 50, 1025 (1983). 
[33] A. Shank, Y. Wang, A. Kaledin, B. Braams, and J. M. Bowman, J. Chem. Phys. 130, 144314 (2009)

[34] R. N. Zare, Angular Momentum (Wiley: New York 1988).

[35] C. Leforestier J. Chem. Phys. 101, 7357 (1994)

[36] Xiao-Gang Wang, Tucker Carrington Jr, Jian Tang, and A. R. W. McKellar J. Chem. Phys. 123, $034301(2005)$

[37] P. Sarkar, N. Poulin, and T. Carrington, J. Chem. Phys. 110, 10269 (1999)

[38] X.-G. Wang and T. Carrington J. Chem. Phys. 138,104106 (2013)

[39] R. A. Toth, J. Mol. Spectrosc. 162, 20 (1993).

[40] H. Wei and T. Carrington, Jr., J. Chem. Phys. 97, 3029 (1992).

[41] J. Echave and D. C. Clary, Chem. Phys. Lett. 190, 225 (1992).

[42] X.-G. Wang and T. Carrington, Jr., J. Chem. Phys. 134, 044313 (2011)

[43] X.-G. Wang and T. Carrington, Jr., J. Chem. Phys. 114, 1473(2001)

[44] R. Chen and H. Guo, J. Chem. Phys. 114, 1467 (2001)

[45] L. H. Coudert and J. T. Hougen, J. Mol. Spectrosc. 130, 86 (1988).

[46] F. N. Keutsch, Thesis, Chemistry (University of California, Berkeley, 2001). 
TABLE I: The minium structure of the D-bonded (HDO-DOH) and the H-bonded (HDO-HOD) isomer of HOD dimer in the dynamical coordinates on the CCpol-8s potential energy surface, with the acceptor on the left and the dimer-fixed frame attached as in Fig. 5. See also Fig. 1.

\begin{tabular}{lcc}
\hline \hline & HDO-DOH (D-bonded) & HDO-HOD (H-bonded) \\
\hline$\left(\alpha_{A e}, \beta_{A e}, \gamma_{A e}\right)(\mathrm{deg})$ & $(0,58.3,104.7)$ & $(0,57.6,105.1)$ \\
$\left(\alpha_{B e}, \beta_{B e}, \gamma_{B e}\right)(\mathrm{deg})$ & $(205.5,82.1,179.2)$ & $(206.5,40.9,-1.2)$ \\
$r_{0 e}(\mathrm{bohr})$ & 5.4419 & 5.5771 \\
\hline \hline
\end{tabular}


TABLE II: $\quad J=0$ energy levels of the D-bonded (HDO-DOH, labelled by D) and the H-bonded (HDO-HOD, labelled by $\mathrm{H}$ ) isomer of HOD dimer $\left(\right.$ in $\left.\mathrm{cm}^{-1}\right)$. "m" means that the state is a mixture of the D-bonded and H-bonded isomers. The second label after the energy is $K$. All the levels up to $200 \mathrm{~cm}^{-1}$ are given. All levels are relative to the ZPE of D-bonded isomer $-1199.2443 \mathrm{~cm}^{-1}$. The ZPE of the H-bonded isomer is $57.3679 \mathrm{~cm}^{-1}$ higher, indicated in bold face.

\begin{tabular}{|c|c|c|c|c|c|c|c|c|c|c|c|c|}
\hline \multirow[b]{2}{*}{1} & \multicolumn{3}{|c|}{$J=0, A^{+}$} & \multicolumn{3}{|c|}{$J=0, B^{+}$} & \multicolumn{3}{|c|}{$J=0, A^{-}$} & \multicolumn{3}{|c|}{$J=0, B^{-}$} \\
\hline & 0.0000 & $\mathrm{D}$ & 0 & 0.0484 & $\mathrm{D}$ & 0 & 7.9243 & $\mathrm{D}$ & 0 & 7.8887 & $\mathrm{D}$ & 0 \\
\hline 2 & 57.3679 & $\mathrm{H}$ & 0 & 57.5360 & $\mathrm{H}$ & 0 & 62.0371 & $\mathrm{H}$ & 0 & 61.8835 & $\mathrm{H}$ & 0 \\
\hline 3 & 59.3109 & $\mathrm{D}$ & 0 & 59.6806 & $\mathrm{D}$ & 0 & 89.3466 & $\mathrm{D}$ & 0 & 88.6759 & $\mathrm{D}$ & 0 \\
\hline 4 & 96.8388 & $\mathrm{D}$ & 0 & 97.0955 & $\mathrm{D}$ & 0 & 101.9852 & $\mathrm{D}$ & 0 & 101.9955 & $\mathrm{D}$ & 0 \\
\hline 5 & 104.5395 & $\mathrm{D}$ & 0 & 106.0817 & $\mathrm{D}$ & 0 & 120.3539 & $\mathrm{D}$ & 0 & 120.2938 & $\mathrm{D}$ & 0 \\
\hline 6 & 111.5243 & $\mathrm{H}$ & 0 & 111.8889 & $\mathrm{H}$ & 0 & 138.1983 & $\mathrm{H}$ & 0 & 138.0850 & $\mathrm{H}$ & 0 \\
\hline 7 & 118.0563 & $\mathrm{D}$ & 0 & 119.7084 & $\mathrm{D}$ & 0 & 148.7331 & $\mathrm{D}$ & 0 & 147.6840 & $\mathrm{D}$ & 0 \\
\hline 8 & 146.8093 & $\mathrm{D}$ & 0 & 146.9133 & $\mathrm{D}$ & 0 & 156.2844 & $\mathrm{H}$ & 0 & 154.7724 & $\mathrm{H}$ & 0 \\
\hline 9 & 149.2467 & $\mathrm{H}$ & 0 & 149.1057 & $\mathrm{H}$ & 0 & 157.8954 & $\mathrm{D}$ & 0 & 156.5962 & $\mathrm{D}$ & 0 \\
\hline 10 & 153.4771 & $\mathrm{H}$ & 0 & 154.6986 & $\mathrm{H}$ & 0 & 159.2012 & $\mathrm{H}$ & 0 & 159.0841 & $\mathrm{H}$ & 0 \\
\hline 11 & 154.4936 & $\mathrm{D}$ & 0 & 162.7574 & $\mathrm{~m}$ & 0 & 169.8538 & $\mathrm{D}$ & 0 & 166.9860 & $\mathrm{D}$ & 0 \\
\hline 12 & 159.5300 & $\mathrm{H}$ & 0 & 164.5240 & $\mathrm{~m}$ & 0 & 185.9490 & $\mathrm{D}$ & 0 & 183.7684 & $\mathrm{D}$ & 0 \\
\hline 13 & 169.5734 & $\mathrm{D}$ & 0 & 170.9740 & $\mathrm{D}$ & 0 & 193.2346 & $\mathrm{D}$ & 0 & 194.5436 & $\mathrm{D}$ & 0 \\
\hline 14 & 183.0715 & $\mathrm{D}$ & 0 & 187.1242 & $\mathrm{D}$ & 0 & 196.4665 & $\mathrm{H}$ & 0 & 197.0753 & $\mathrm{~m}$ & 0 \\
\hline 15 & 186.6550 & $\mathrm{D}$ & 0 & 188.6736 & $\mathrm{D}$ & 0 & & & & 199.3191 & $\mathrm{H}$ & 0 \\
\hline 16 & 194.8086 & $\mathrm{D}$ & 0 & 196.9386 & $\mathrm{~m}$ & 0 & & & & & & \\
\hline 17 & 197.2810 & $\mathrm{~m}$ & 0 & & & 0 & & & & & & \\
\hline
\end{tabular}


TABLE III: Same as Table II for $J=1$. All the levels up to $150 \mathrm{~cm}^{-1}$ are given.

\begin{tabular}{|c|c|c|c|c|c|c|c|c|c|c|c|c|}
\hline \multirow[b]{2}{*}{1} & \multicolumn{3}{|c|}{$J=1, A^{+}$} & \multicolumn{3}{|c|}{$J=1, B^{+}$} & \multicolumn{3}{|c|}{$J=1, A^{-}$} & \multicolumn{3}{|c|}{$J=1, B^{-}$} \\
\hline & 8.0016 & $\mathrm{D}$ & 1 & 8.0483 & $\mathrm{D}$ & 1 & 0.4401 & $\mathrm{D}$ & 0 & 0.3917 & $\mathrm{D}$ & 0 \\
\hline 2 & 8.2861 & $\mathrm{D}$ & 0 & 8.3219 & $\mathrm{D}$ & 0 & 8.0506 & $\mathrm{D}$ & 1 & 8.0037 & D & 1 \\
\hline 3 & 12.3788 & $\mathrm{D}$ & 1 & 12.4141 & $\mathrm{D}$ & 1 & 12.4156 & $\mathrm{D}$ & 1 & 12.3804 & $\mathrm{D}$ & 1 \\
\hline 4 & 62.2583 & $\mathrm{H}$ & 0 & 62.4118 & $\mathrm{H}$ & 0 & 57.9113 & $\mathrm{H}$ & 0 & 57.7433 & $\mathrm{H}$ & 0 \\
\hline 5 & 64.0333 & $\mathrm{H}$ & 1 & 64.1556 & $\mathrm{H}$ & 1 & 60.0724 & $\mathrm{D}$ & 0 & 59.7033 & $\mathrm{D}$ & 0 \\
\hline 6 & 65.6934 & $\mathrm{H}$ & 1 & 65.8463 & $\mathrm{H}$ & 1 & 64.1532 & $\mathrm{H}$ & 1 & 64.0309 & $\mathrm{H}$ & 1 \\
\hline 7 & 73.3259 & $\mathrm{D}$ & 1 & 73.6480 & $\mathrm{D}$ & 1 & 65.8467 & $\mathrm{H}$ & 1 & 65.6938 & $\mathrm{H}$ & 1 \\
\hline 8 & 82.8333 & $\mathrm{D}$ & 1 & 83.4834 & $\mathrm{D}$ & 1 & 73.6454 & $\mathrm{D}$ & 1 & 73.3231 & $\mathrm{D}$ & 1 \\
\hline 9 & 89.0725 & $\mathrm{D}$ & 0 & 89.7425 & $\mathrm{D}$ & 0 & 83.4828 & $\mathrm{D}$ & 1 & 82.8327 & $\mathrm{D}$ & 1 \\
\hline 10 & 98.9965 & $\mathrm{D}$ & 1 & 99.4370 & $\mathrm{D}$ & 1 & 97.4850 & $\mathrm{D}$ & 0 & 97.2285 & D & 0 \\
\hline 11 & 102.3879 & $\mathrm{D}$ & 0 & 102.3777 & $\mathrm{D}$ & 0 & 99.4378 & $\mathrm{D}$ & 1 & 98.9972 & $\mathrm{D}$ & 1 \\
\hline 12 & 110.6856 & $\mathrm{D}$ & 1 & 110.9293 & $\mathrm{D}$ & 1 & 106.4749 & $\mathrm{D}$ & 0 & 104.9347 & $\mathrm{D}$ & 0 \\
\hline 13 & 120.6851 & $\mathrm{D}$ & 0 & 120.7452 & $\mathrm{D}$ & 0 & 110.9281 & $\mathrm{D}$ & 1 & 110.6843 & $\mathrm{D}$ & 1 \\
\hline 14 & 121.1576 & $\mathrm{D}$ & 1 & 121.9653 & $\mathrm{D}$ & 1 & 112.2630 & $\mathrm{H}$ & 0 & 111.8987 & $\mathrm{H}$ & 0 \\
\hline 15 & 122.4667 & $\mathrm{D}$ & 1 & 122.9186 & $\mathrm{D}$ & 1 & 120.0991 & $\mathrm{D}$ & 0 & 118.4489 & $\mathrm{D}$ & 0 \\
\hline 16 & 126.4415 & $\mathrm{H}$ & 1 & 126.4528 & $\mathrm{H}$ & 1 & 121.9692 & $\mathrm{D}$ & 1 & 121.1623 & $\mathrm{D}$ & 1 \\
\hline 17 & 127.8303 & $\mathrm{H}$ & 1 & 128.1307 & $\mathrm{H}$ & 1 & 122.9213 & $\mathrm{D}$ & 1 & 122.4685 & $\mathrm{D}$ & 1 \\
\hline 18 & 138.4627 & $\mathrm{H}$ & 0 & 138.5759 & $\mathrm{H}$ & 0 & 126.4516 & $\mathrm{H}$ & 1 & 126.4403 & $\mathrm{H}$ & 1 \\
\hline 19 & 142.1464 & $\mathrm{D}$ & 1 & 143.7149 & $\mathrm{D}$ & 1 & 128.1285 & $\mathrm{H}$ & 1 & 127.8280 & $\mathrm{H}$ & 1 \\
\hline 20 & 143.5533 & $\mathrm{D}$ & 1 & 146.8428 & $\mathrm{D}$ & 1 & 143.7165 & $\mathrm{D}$ & 1 & 142.1432 & $\mathrm{D}$ & 1 \\
\hline 21 & 148.0756 & $\mathrm{D}$ & 0 & 149.1215 & $\mathrm{D}$ & 0 & 146.8422 & $\mathrm{D}$ & 1 & 143.5549 & $\mathrm{D}$ & 1 \\
\hline 22 & & & & & & & 147.2930 & $\mathrm{D}$ & 0 & 147.1882 & $\mathrm{D}$ & 0 \\
\hline 23 & & & & & & & 149.4814 & $\mathrm{H}$ & 0 & 149.6213 & $\mathrm{H}$ & 0 \\
\hline
\end{tabular}


TABLE IV: The AS tunnelling splitting $a(K)\left(\mathrm{in}^{-1}\right)$ for the D-bonded (HDO-DOH) isomer of HOD dimer

\begin{tabular}{rrrr}
\hline \hline$a(K=0)$ & obs. $^{a}$ & cal. $(J=0)$ & cal. $(J=1)$ \\
\hline & 7.145 & 7.882 & 7.888 \\
& & & \\
\hline$a(K=1)$ & obs. $^{a}$ & cal. $(K=-1)$ & cal. $(K=+1)$ \\
\hline \hline \multicolumn{4}{c}{ a Ref. 30.}
\end{tabular}

TABLE V: The interchange tunnelling splitting $i_{v, v^{\prime}}\left(\right.$ in $\left.\mathrm{cm}^{-1}\right)$ for the D-bonded (HDO-DOH) isomer of HOD dimer

\begin{tabular}{lrrr}
\hline \hline & obs. $^{a}$ & cal. $(J=0)$ & cal. $(J=1)$ \\
\hline$i_{0,1}$ & 0.0441 & 0.0484 & 0.0484 \\
$i_{2,3}$ & 0.0335 & 0.0356 & 0.0358 \\
& & & \\
\hline$i_{4,5}$ & obs. $^{a}$ & cal. $(K=-1)$ & cal. $(K=+1)$ \\
$i_{6,7}$ & 0.0422 & 0.0469 & 0.0467 \\
\hline \hline
\end{tabular}

${ }^{a}$ Ref. 30 . 
TABLE VI: The AS tunnelling splitting $a(K)\left(\mathrm{in}^{-1}\right)$ for the H-bonded (HDO-DOH) isomer of HOD dimer

\begin{tabular}{|c|c|c|c|}
\hline & obs. $^{a}$ & cal. $(J=0)$ & cal. $(J=1)$ \\
\hline \multirow[t]{2}{*}{$a(K=0)$} & 3.917 & 4.508 & 4.507 \\
\hline & obs. $^{a}$ & cal. $(K=-1)$ & $\operatorname{cal} .(K=+1)$ \\
\hline$a(K=1)$ & 1.519 & 1.676 & 1.678 \\
\hline
\end{tabular}

TABLE VII: The interchange tunnelling splitting $i_{v, v^{\prime}}\left(\right.$ in $\left.\mathrm{cm}^{-1}\right)$ for the H-bonded (HDO-DOH) isomer of HOD dimer

\begin{tabular}{lrrr}
\hline \hline & obs. $^{a}$ & cal. $(J=0)$ & cal. $(J=1)$ \\
\hline$i_{0,1}$ & 0.1669 & 0.1681 & 0.1700 \\
$i_{2,3}$ & 0.1543 & 0.1536 & 0.1535 \\
& & & \\
\hline$i_{4,5}$ & obs. $^{a}$ & cal. $(K=-1)$ & cal. $(K=+1)$ \\
$i_{6,7}$ & 0.1250 & 0.1223 & 0.1223 \\
\hline \hline
\end{tabular}

${ }^{a}$ Ref. 30 . 


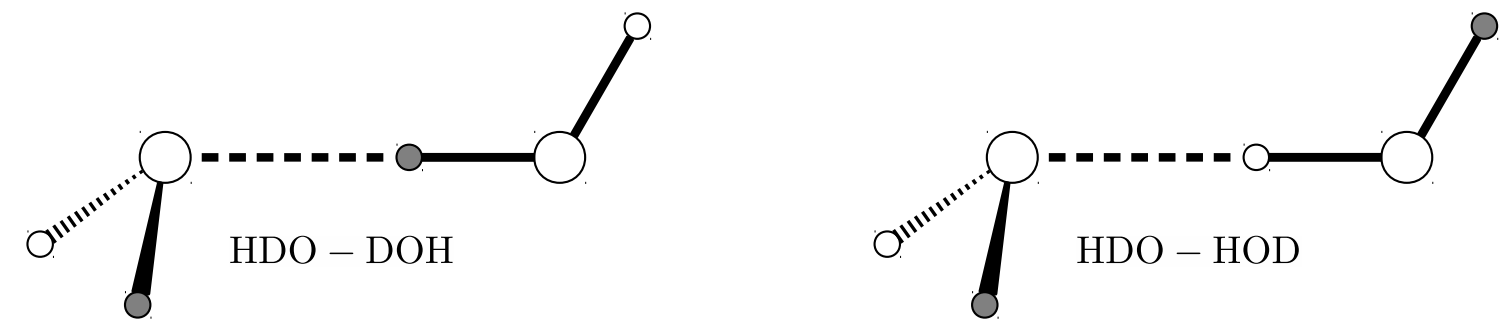

FIG. 1: Two isomers of HOD dimer: D-bonded (HDO-DOH) isomer on the left, H-bonded (HDOHOD) isomer on the right.
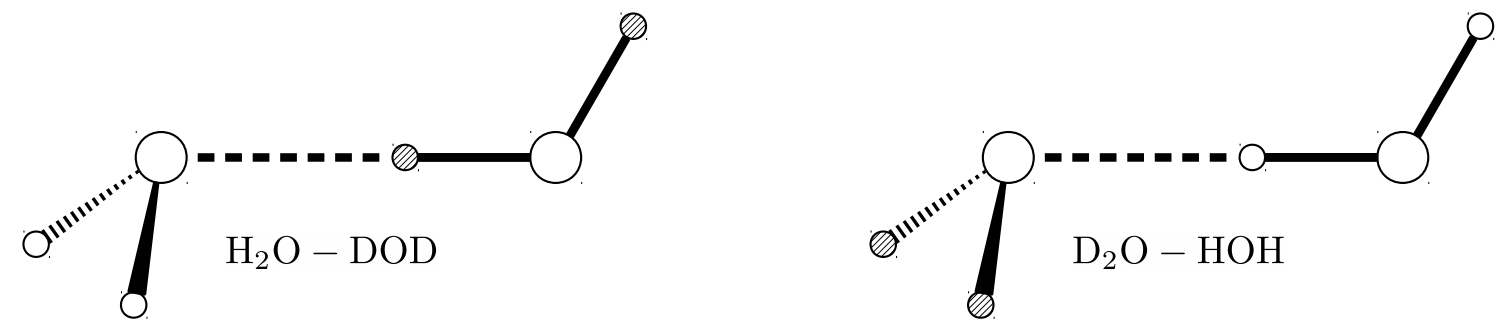

FIG. 2: Two isomers of $\mathrm{H}_{2} \mathrm{O}-\mathrm{D}_{2} \mathrm{O}$.
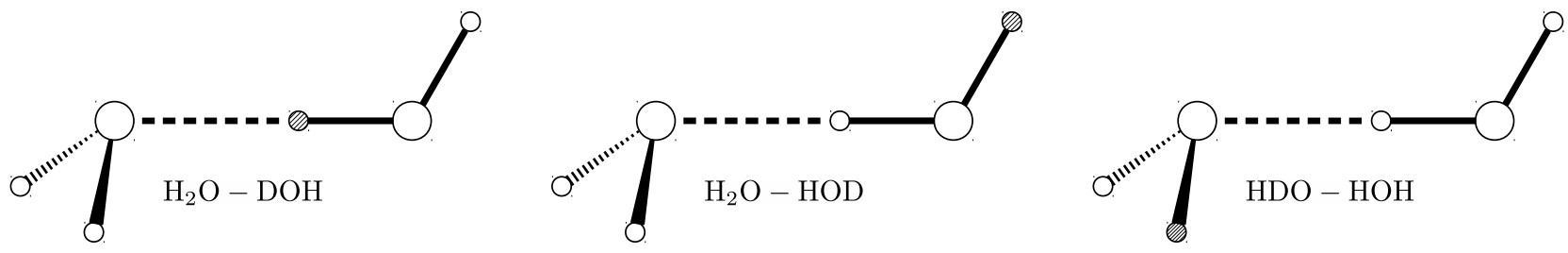

FIG. 3: Three isomers of $\mathrm{H}_{2} \mathrm{O}-\mathrm{HDO}$. 

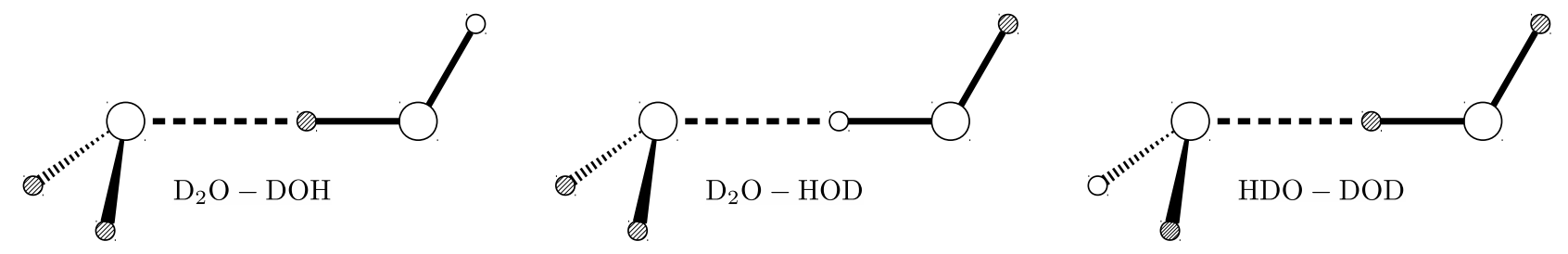

FIG. 4: Three isomers of $\mathrm{D}_{2} \mathrm{O}-\mathrm{HDO}$. 


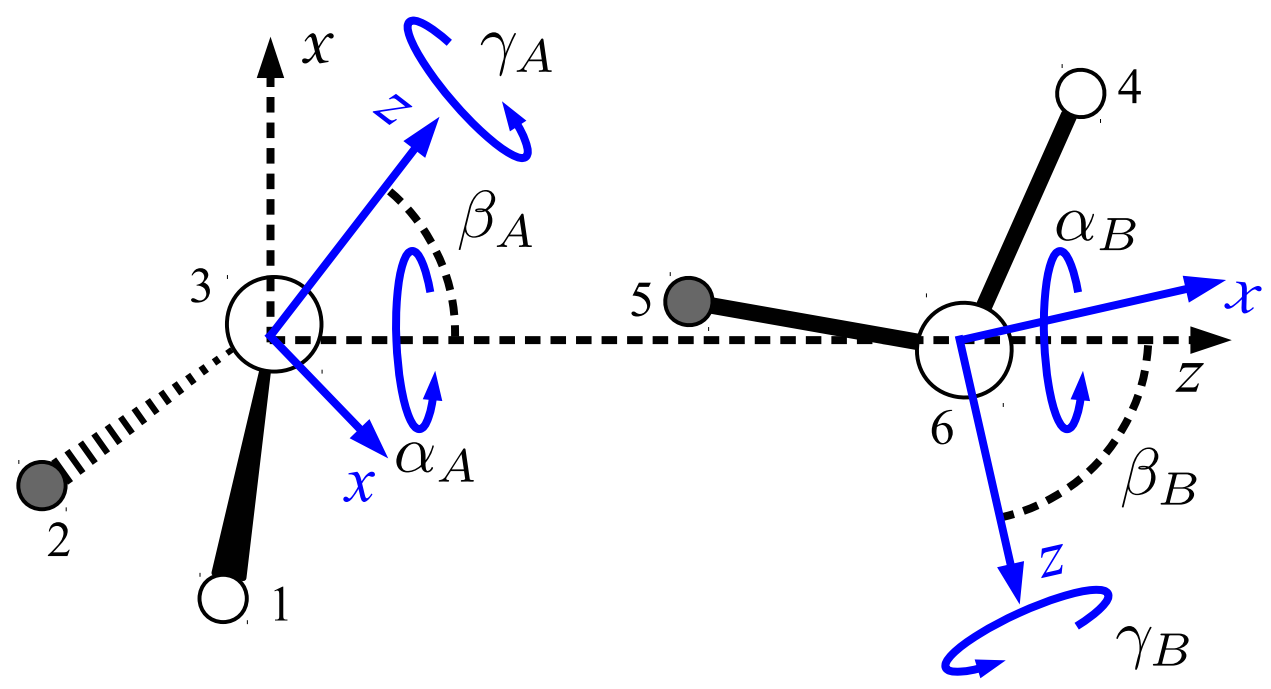

(a)

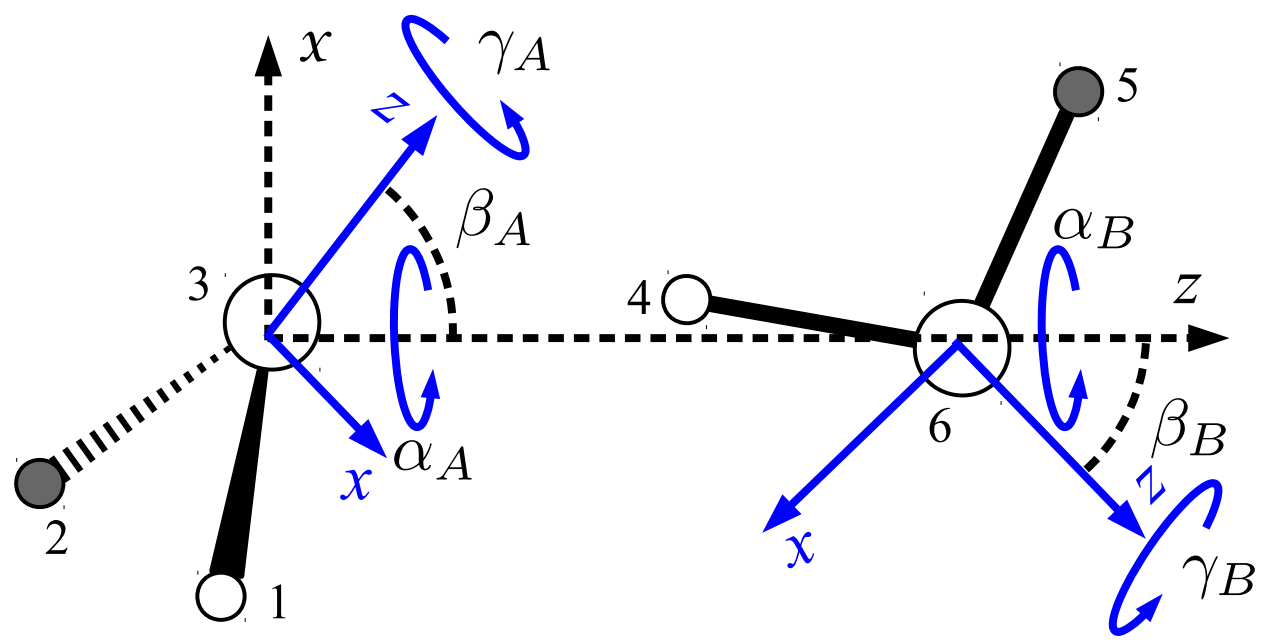

(b)

FIG. 5: The angles defining the MF and DF frames for the D-bonded (HDO-DOH) isomer, panel (a), and the H-bonded (HDO-HOD) isomer, panel (b) of HOD dimer. The DF frame is indicated by dashed $x-z$ axes. The two MF frames are indicated by blue $x-z$ axes. The A MF frame is the principal axes frame with the $z$-axis along the $b$-axis and such that the $z$ coordinate of atom 3 is positive, and the $x$-axis in the HDO plane and such that the $x$ coordinate of atom 1 of monomer is positive. The D-atom is black. With the acceptor at the left, labelled as $A$, and donor on the right, labelled as $B$, the equilibrium coordinates are: $\left(\alpha_{A}, \beta_{A}, \gamma_{A} ; \alpha_{B}, \beta_{B}, \gamma_{B}\right)=\left(0.0^{\circ}, 58.3^{\circ}, 104.7^{\circ} ; 205.5^{\circ}, 82.1^{\circ}, 179.2^{\circ}\right) ; r_{0 e}=5.4419 a_{0}$, for the $\mathrm{D}$ bonded isomer; and $\left(\alpha_{A}, \beta_{A}, \gamma_{A} ; \alpha_{B}, \beta_{B}, \gamma_{B}\right)=\left(0.0^{\circ}, 57.6^{\circ}, 105.1^{\circ} ; 206.5^{\circ}, 40.9^{\circ},-1.2^{\circ}\right) ; r_{0 e}=$ $5.5771 a_{0}$, for the H-bonded isomer. 




FIG. 6: The MF frame $(x-z)$ for HOD and the MF frame $\left(x^{\prime}-z^{\prime}\right)$ for $\mathrm{H}_{2} \mathrm{O}$. The HDO MF frame is tilted with respect to the $\mathrm{H}_{2} \mathrm{O}$ MF frame by $21.0^{\circ}$. The origin of the $\mathrm{H}_{2} \mathrm{O}$ MF frame is shifted with respect to the origin of HDO MF frame. Atom 1 is $\mathrm{H}$ and atom 2 is D. 


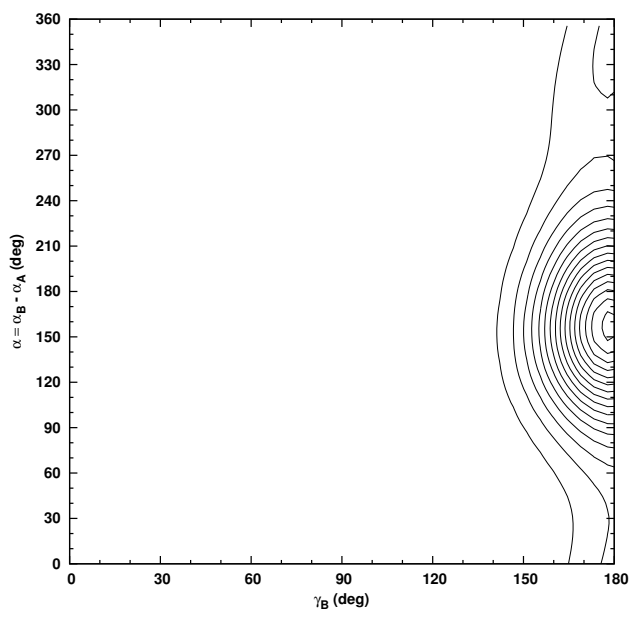

(a)

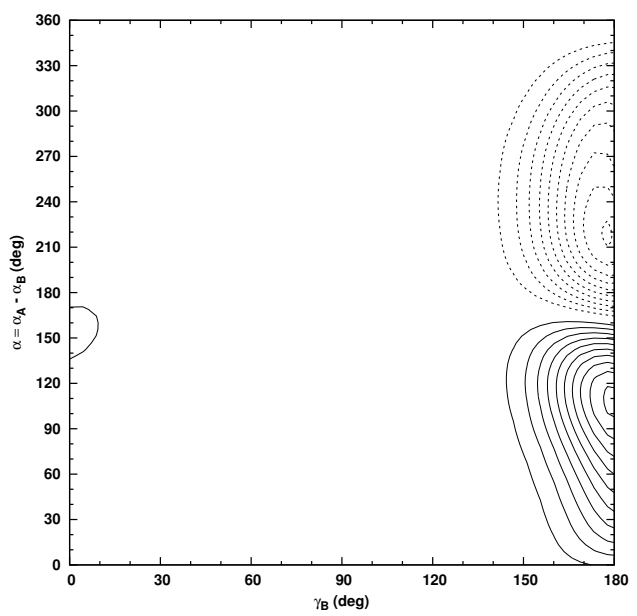

(c)

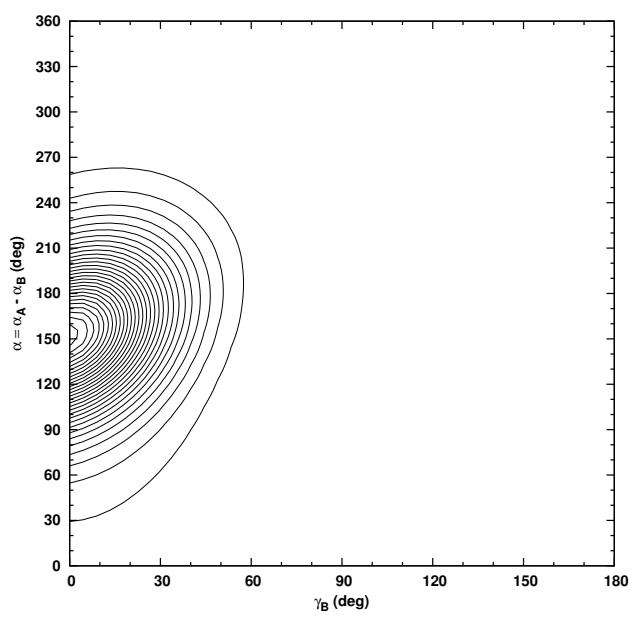

(b)

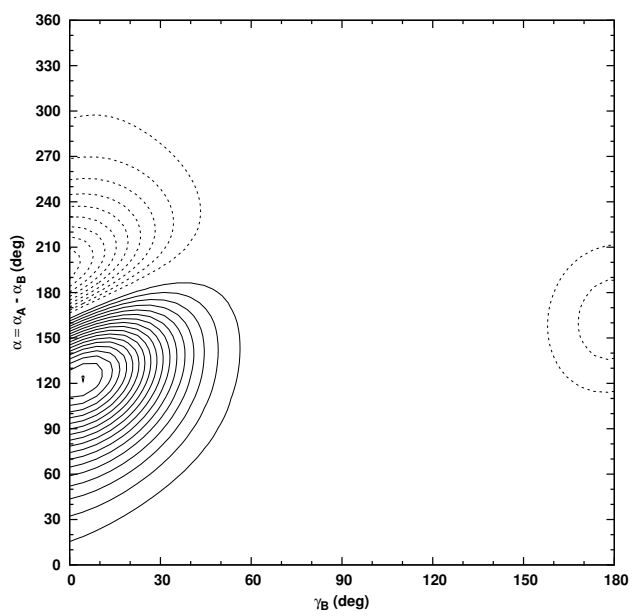

(d)

FIG. 7: Wavefunction cuts for the D-bonded isomer: ground state at $0 \mathrm{~cm}^{-1}\left(A^{+}\right)$(a) and first excited state at $59.31 \mathrm{~cm}^{-1}\left(A^{+}\right)(\mathrm{c})$. Wavefunction cuts for the H-bonded isomer: ground state at $57.37 \mathrm{~cm}^{-1}\left(A^{+}\right)(\mathrm{b})$ and first excited state at $111.52 \mathrm{~cm}^{-1}\left(A^{+}\right)(\mathrm{d})$. 


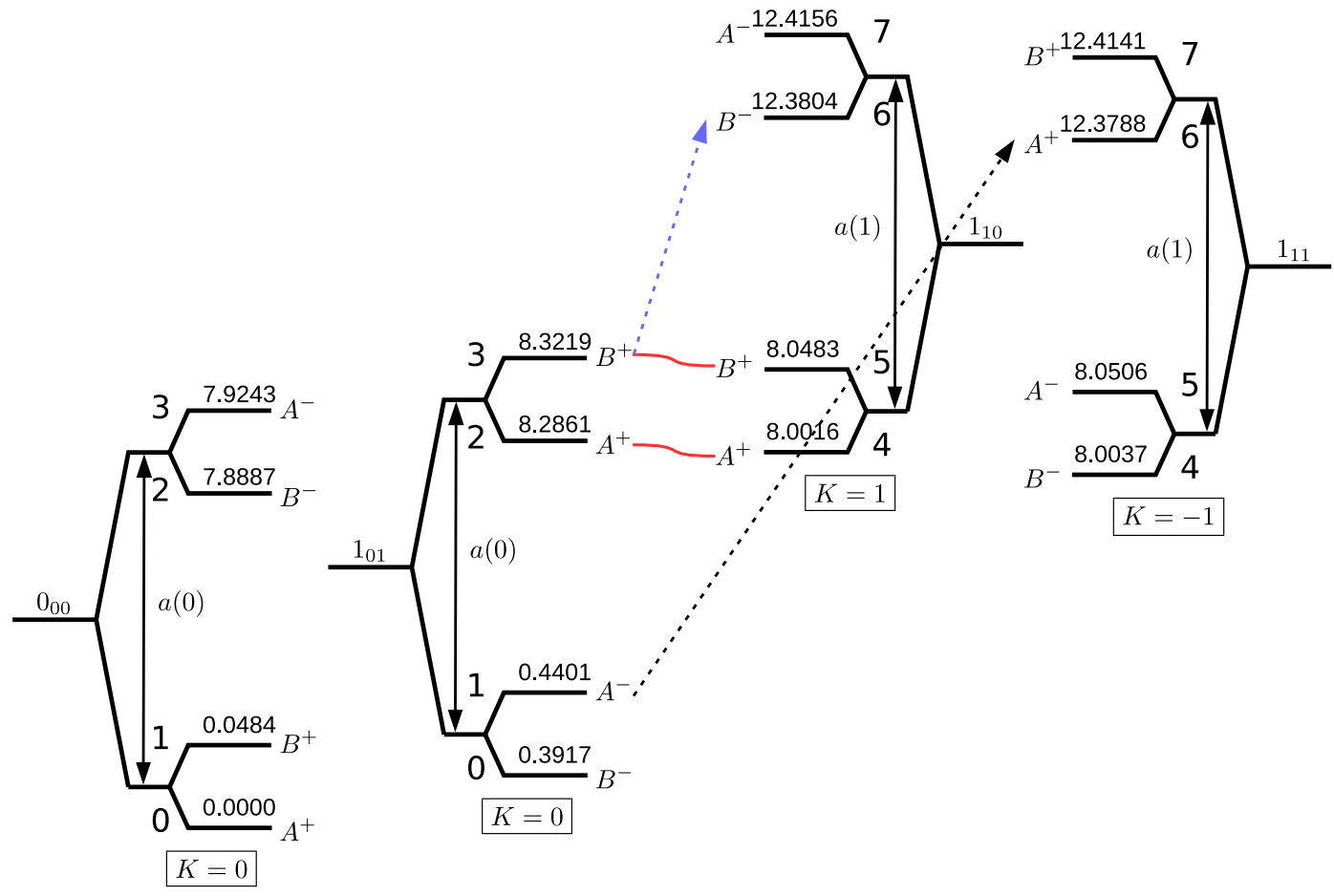

FIG. 8: Schematic diagram of $J=0$ and $J=1$ levels of the D-bonded isomer of HOD dimer [45]. Each level is labelled by a value of $\mathrm{V}$ (see the text). Two observed transitions are indicated: a b-type transition (in blue) between $V=3$ and 6 at $104.828 \mathrm{GHz}$, and a c-type transition (in black) between $V=1$ and 6 at $222.054 \mathrm{GHz}$ for $\mathrm{H}$-bonded isomer. The difference of these two transitions could be used to determine $a(0)$. Values of the calculated $J=0$ and $J=1$ levels of the D-bonded isomer of HOD dimer are also indicated. 


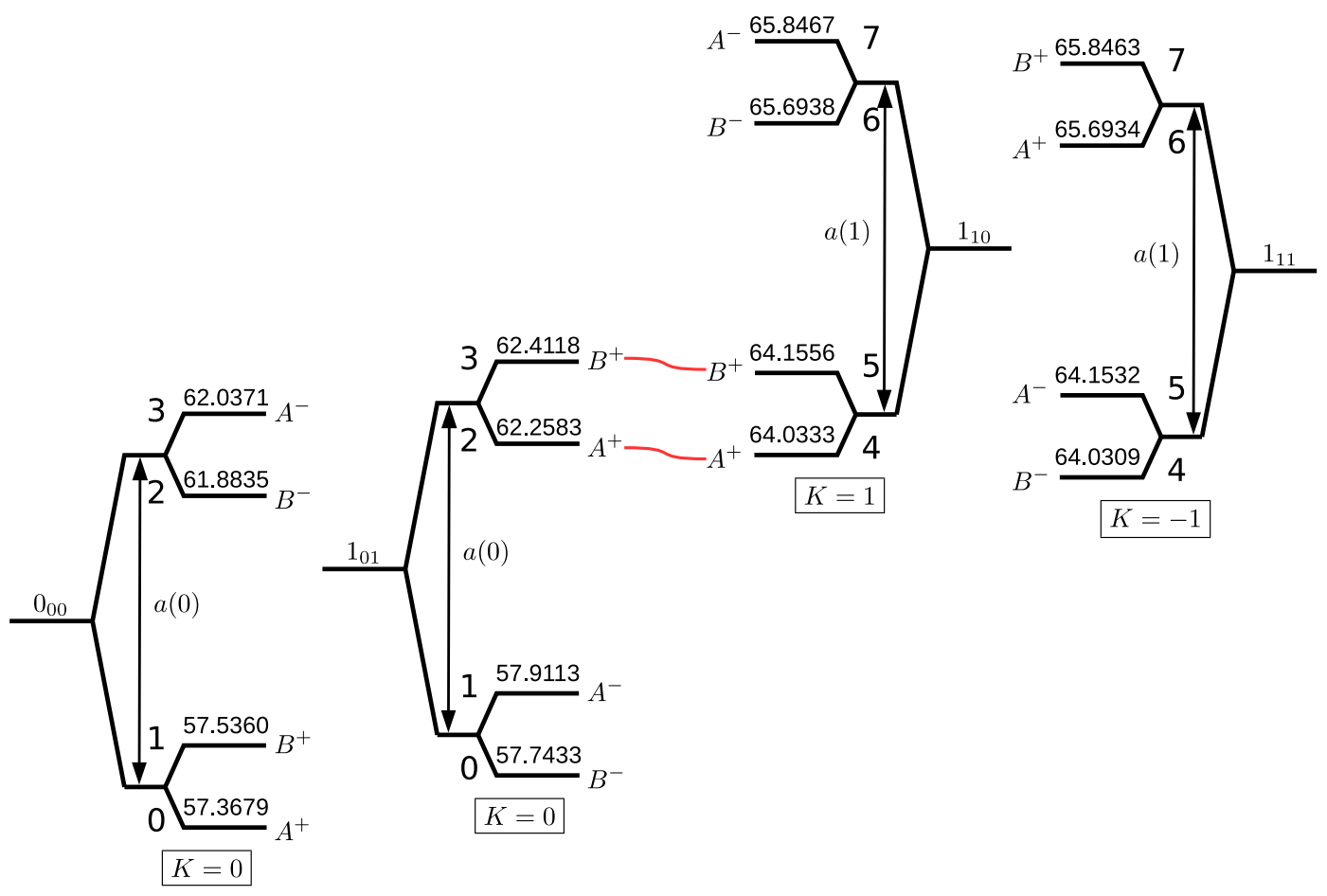

FIG. 9: All calculated $J=0$ and $J=1$ levels of H-bonded isomer of HOD dimer. 